\title{
Matrilysin (MMP-7) is a major matrix metalloproteinase upregulated in biliary atresia-associated liver fibrosis
}

\author{
Chao-Cheng Huang ${ }^{1,2}$, Jiin-Haur Chuang ${ }^{1,3,4}$, Ming-Huei Chou ${ }^{1}$, Chia-Lin $\mathrm{Wu}^{3}$, \\ Ching-Mei Chen ${ }^{2}$, Chih-Chi Wang ${ }^{4}$, Yaw-Sen Chen ${ }^{4}$, Chao-Long Chen ${ }^{4}$ and Ming-Hong Tai ${ }^{5}$ \\ ${ }^{1}$ Graduate Institute of Clinical Medical Sciences, Chang Gung University, Lin-Ko, Taiwan; ${ }^{2}$ Department of \\ Pathology, Chang Gung Memorial Hospital, Kaohsiung, Taiwan; ${ }^{3}$ Department of Pediatric Surgery, Chang \\ Gung Memorial Hospital, Kaohsiung, Taiwan; ${ }^{4}$ Department of Surgery, Chang Gung Memorial Hospital, \\ Kaohsiung, Taiwan and ${ }^{5}$ Department of Education and Research, Veterans General Hospital, Kaohsiung, \\ Taiwan
}

\begin{abstract}
Matrix metalloproteinases (MMPs) are the proteases responsible for tissue remodeling during liver fibrosis caused by various disorders including biliary atresia. However, information regarding the relative contribution of these proteases to liver fibrosis is still limited. We studied matrix metalloproteinase-2 (MMP-2), -7, -9 and -13 mRNA expressions in the liver tissue of early-stage biliary atresia at the time of Kasai's procedure, late-stage biliary atresia at the time of liver transplantation with advanced fibrosis and nondiseased control without liver fibrosis. The results of real-time quantitative reverse transcriptase-PCR analysis revealed that only MMP-2 and -7 expressions were significantly different between groups. MMP-2 was significantly higher in Liver Transplantation group than both in Control $(P=0.010)$ and in Kasai's Procedure $(P=0.001)$ groups, whereas the difference of MMP-2 expression between Control and Kasai's Procedure was not significant. However, the relative expression level of MMP-7 was sequentially elevated when comparing Control, Kasai's Procedure and Liver Transplantation groups, and there was significant $(P=0.019)$ difference when comparing Control and Liver Transplantation groups. Moreover, the fold difference in MMP-7 mRNA was much higher than that in MMP-2 mRNA between groups. The expressions of MMP-7 were further confirmed by agarose gel electrophoresis and Western blotting. Immunohistochemical analysis revealed a significant positive correlation of the scores of MMP-7 immunostaining with the stages of liver fibrosis. In situ hybridization demonstrated that the bile ductular epithelial cells, Kupffer cells and hepatocytes were the major producers of matrix metalloproteinase-7 in the liver. Our results imply that MMP-7 is a major MMP associated with the tissue remodeling during the progression of liver fibrosis in biliary atresia.

Modern Pathology (2005) 18, 941-950. doi:10.1038/modpathol.3800374; Published online 28 January 2005
\end{abstract}

Keywords: biliary atresia; liver fibrosis; matrix metalloproteinases; matrilysin; quantitative RT-PCR; in situ hybridization

It has recently been suggested that biliary atresia is not a single disease, but a phenotype of several underlying specific disorders to which the infant responds in a stereotypic manner by a complex dynamic process. ${ }^{1}$ The grave prognosis in biliary atresia invariably depends on the occurrence of liver cirrhosis, which is currently the leading cause of liver transplantation in children. ${ }^{2-4}$ Kasai's porto-

Correspondence: Dr J-H Chuang, MD, Department of Pediatric Surgery, Chang Gung Memorial Hospital, 123 Ta-Pei Road, Niao-Sung Hsiang, Kaohsiung County 833, Taiwan.

E-mail: jhchuang@adm.cgmh.org.tw

Received 27 May 2004; revised and accepted 8 December 2004; published online 28 January 2005 enterostomy is the only effective procedure for correction of biliary atresia at the early stage. However, many of the patients who have received the Kasai's procedure still develop liver cirrhosis even though the bile flow is adequate. ${ }^{5}$ Hence, liver fibrosis is still a major problem in the treatment of biliary atresia.

The progress of liver cirrhosis is a complex process involving many cytokines related to activation of the hepatic stellate cells and alteration of the extracellular matrix. ${ }^{6-12}$ The extracellular matrix holds cells together and maintains the three-dimensional structure of organs including the liver. The extracellular matrix component of the normal liver is relatively minor, and liver fibrosis is the result of 
extensive tissue remodeling with a net increase in extracellular matrix. The key enzymes responsible for degradation and deposition of all the protein components of extracellular matrix and basement membrane are matrix metalloproteinases (MMPs) and their endogenous inhibitors. There have been 24 human MMPs and four tissue inhibitors of metalloproteinases (TIMPs) identified. ${ }^{13}$ It is known that most of MMPs are not constitutively expressed in the normal liver. ${ }^{14}$ Many studies have focused on the expression profiles of MMPs in liver fibrosis, but the results are inconsistent and obviously affected by their underlying diseases and the different methods of detection. ${ }^{15-22}$

To address the major MMPs that involve in the progress of liver fibrosis in biliary atresia, we studied $M M P-2, M M P-9$ and $M M P-13$ that were most commonly reported MMPs related to the regulation of liver fibrogenesis. We also studied MMP-7, which has been mentioned being the key regulator of pulmonary fibrosis ${ }^{23}$ and is expressed in significantly higher level in DNA microarray ${ }^{24,25}$ but not being studied otherwise in liver fibrosis associated with biliary atresia. Real-time quantitative reverse transcriptase-PCR (qRT-PCR) showed a significantly higher expression of $M M P-2$ and $M M P-7$ in the late cirrhotic stage of liver compared to the control. However, the fold difference in the expression of $M M P-7$ mRNA was much higher than that of MMP-2 mRNA. These findings were consistent with the results of MMP-7 protein expression studies by Western blotting and by immunohistochemistry, and implied that MMP-7 is a major MMP involving in the progress of liver fibrosis associated with biliary atresia.

\section{Materials and methods}

\section{Patients and Samples}

To compare MMP-2, -7, -9 and -13 expression between early and late stages of biliary atresia, 30 snap frozen liver samples were analyzed with realtime qRT-PCR. A total of 12 wedge liver biopsy samples of early biliary atresia patients receiving Kasai's procedure were referred as the Kasai's Procedure group, and 12 liver explants of late biliary atresia patients receiving liver transplantation were referred as the Liver Transplantation group. The other six nondiseased liver samples, referred as the Control group, were derived from the young children receiving partial resection of liver in abdominal operations without any association with biliary disorders. Five of them were removed from patients who underwent repairs of hiatus hernia and diaphragmatic hernia, corrections of malrotation and esophageal atresia, and closure of gastrostomy, respectively. The other one was obtained from partial hepatectomy for internal bleeding. All the liver tissues were used after the informed consent was obtained from the patients' parents. There were seven female and five male patients in the Liver Transplantation group, six female and six male patients in the Kasai's Procedure group, and two female and four male subjects in the Control group. The mean age of these patients in Liver Transplantation, Kasai's Procedure and Control groups was $15 \pm 6,2 \pm 1$ and $18 \pm 24$ months, respectively.

To correlate the MMP-7 immunochemical staining with the stages of liver fibrosis, the archival liver tissues of 57 patients with biliary atresia, including 36 in Kasai's procedure and 21 in liver transplantation from the Department of Pathology, Chang Gung Memorial Hospital, Kaohsiung, were examined and staged for liver fibrosis as suggested by Ferrell ${ }^{26}$ after Masson's trichrome staining. All the liver specimens for qRT-PCR were also included. The study, including the use of fresh and paraffin-embedded tissue specimens, was approved by the Medical Ethics and the Human Clinical Trial Committee at Chang Gung Memorial Hospital, Taiwan.

\section{RNA Isolation and qRT-PCR}

Real-time qRT-PCR was performed using the ABI 7700 Sequence Detection System (Perkin-Elmer Life Sciences, Boston, MA, USA). Total RNA was isolated from frozen liver tissue using a single-step method with REZOL ${ }^{\mathrm{TM}} \mathrm{C} \& \mathrm{~T}$ (Protech technology, Taipei, Taiwan), and the first-strand complementary DNA (cDNA) was synthesized by reverse transcription with oligo(dT)15 primers, M-MLV reverse transcriptase, and dNTP mixture (Promega, Madison, WI, USA). PCR was performed in $50 \mu \mathrm{l}$ SYBR Green PCR Master Mix (Applied Biosystems, Foster City, CA, USA) containing $25 \mu \mathrm{l}$ of the SYBR Green PCR master mix, $0.2 \mu \mathrm{M}$ each of forward and reverse primers, and approximately $30 \mathrm{ng}$ of cDNA. The sequence of the primers used in this study was shown in Table 1.

Amplification and detection was performed with the following profile: 1 cycle of $95^{\circ} \mathrm{C}$ for $10 \mathrm{~min}$ and 40 cycles of $95^{\circ} \mathrm{C}$ for $15 \mathrm{~s}, 60^{\circ} \mathrm{C}$ for $30 \mathrm{~s}$, and $72^{\circ} \mathrm{C}$ for $15 \mathrm{~s}$. After amplification, a final melting curve was performed according to the dissociation protocol of the ABI7700 instrument. Real-time fluorescence measurement was read and a threshold cycle $\left(C_{\mathrm{T}}\right)$ value for each sample was calculated by determining the point at which the fluorescence exceeded a threshold limit, that is, 10 times the standard deviation of the baseline. $\beta$-Actin was also quantified as the endogenous RNA control. Each sample was normalized to its $\beta$-actin content. Relative quantitation of gene expression was based on comparative $C_{\mathrm{T}}$ method, in which the amount of target was given by $2^{-\left(\Delta C_{\mathrm{T}} \text { target }-\Delta C_{\mathrm{T}} \text { calibrator }\right)}$ or $2^{-\Delta \Delta C_{\mathrm{T}}}$. The calibrator was chosen as it had the lowest expression value across the groups for the same gene under statistical comparison. To confirm the specificity of the PCR reaction, PCR products were electrophoresed on a $1.5 \%$ agarose gel. 
Table 1 Primer sequences of MMPs and $\beta$-actin

\begin{tabular}{lll}
\hline Cytokines & Forward primers & Reverse primers \\
\hline$M M P s$ & & \\
$M M P-2$ & $5^{\prime}$-CAACTACGATGATGACCGCAA-3' & $5^{\prime}$-GTGTAAATGGGTGCCATCAGG-3' $^{\prime}$ \\
$M M P-7$ & $5^{\prime}$-TACAGTGGGAACAGGCTCAGG-3' & $5^{\prime}$-GGCACTCCACATCTGGGCT-3' $^{\prime}$ \\
$M M P-9$ & $5^{\prime}$-GACCTGGGCAGATTCCAAAC-3' & $5^{\prime}$-CACGCGCAGTGAAGGTGAGC-3' \\
$M M P-13$ & $5^{\prime}$-CTTCACGATGGCATTGCTGA-3' & $5^{\prime}$-AACTCATGCGCAGCAACAAG-3' \\
Human $\beta$-actin & & \\
& $5^{\prime}$-TCACCCACACTGTGCCCATCTACG-3' & $5^{\prime}$-CAGCGGAACCGCTCATTGCCAATGG-3'
\end{tabular}

\section{Western Blotting}

To identify MMP-7 protein in liver tissue, rabbit polyclonal antibody Ab-4 recognizing both proenzyme and active enzyme of MMP-7 and mouse monoclonal antibody Ab-2 recognizing active form of MMP-7 only were purchased (Oncogene Research Products, Boston, MA, USA). Total proteins from two fresh liver specimens each of the Control, Kasai's Procedure and Liver Transplantation groups were isolated respectively by protein extraction reagent (Pierce Biotechnology, Rockford, IL, USA). The amount of total protein was measured with Bio-Rad protein assay method (Bio-Rad Laboratories, Hercules, CA, USA). In all, $50 \mu \mathrm{g}$ of total protein of each sample was separated by SDS-PAGE using a $12.5 \%$ gel. The separated proteins were transferred and immobilized from gel to a PVDF membrane. To block nonspecific reactivity, the membranes were treated with phosphate-buffered saline (PBS) buffer containing 3\% dry milk for $1 \mathrm{~h}$. Then either MMP-7 Ab-4 or Ab-2 antibody was applied for $2 \mathrm{~h}$. After PBS washing, the membrane reacted with MMP-7 Ab-4 was incubated with horseradish peroxidase (HRP)-linked goat anti-rabbit antibody, and that reacted with MMP-7 Ab-2 was incubated with HRP-linked goat anti-mouse antibody (Santa Cruz Biotechnology, Santa Cruz, CA, USA), respectively, for $1 \mathrm{~h}$. Detection was carried out with the ECL Western blotting kit (Amersham Biosciences, Bucks, UK). The amount of $\alpha$-tubulin was also detected as the internal control.

\section{Immunohistochemistry}

Liver sections (2- $\mu \mathrm{m}$ thick) on poly-L-lysine-coated slides were dewaxed, treated with $3 \%$ hydrogen peroxide for $10 \mathrm{~min}$ to inactivate the endogenous peroxidase activity and microwaved for $10 \mathrm{~min}$ in $10 \mathrm{mM}$ citrate buffer to retrieve antigens. The sections were then incubated with rabbit MMP-7 polyclonal antibody Ab-4 (1:100 dilution) for $1 \mathrm{~h}$ at room temperature. After washing with PBS, the sections were reacted with the HRP/Fab polymer conjugate (PicTure ${ }^{\mathrm{TM}}$-Plus kit) (Zymed, South San Francisco, CA, USA) according to the manufacturer's instructions and then incubated with $\mathrm{DAB}$ chromogen. The sections were finally counter stained with Gill's hematoxylin (Merck, Darmstadt, Germany). The paraffin sections of infiltrating duct carcinoma of breast were used as positive control by the recommendation of the manufacturer. Negative control was also done by omitting the primary antibody. The immunoreactivity was scored using an arbitrary scale of 0 to $3+$ reflecting the intensity of corresponding immunoreactive MMP-7.

\section{Non-Radioactive In Situ Hybridization}

To localize MMP-7 gene in the liver tissue, we used a protocol of nonradioactive in situ hybridization (ISH) with catalyzed reporter deposition method for signal amplification as previously described. ${ }^{27,28}$ For preparation of the probes, the cDNA was obtained and subcloned into pGEM ${ }^{\mathbb{R}}$-T Easy Vector. Digoxigenin-labeled antisense riboprobes were synthesized using in vitro transcription method according to the manufacturer's instructions (Roche, Mannheim, Germany). The sense riboprobes were also synthesized by the same method to be served as the negative control in ISH. The cohort sections were prepared by cutting the paraffin-embedded tissue blocks to $4 \mu \mathrm{m}$ in thickness and placing on the silane coating slides followed by deparaffinization. The procedure of deparaffinizaion and rehydration of the sections was handled under RNase-free condition with the use of diethylpyrocarbonate water. The sections were then digested with 20 $\mu \mathrm{g} / \mathrm{ml}$ proteinase $\mathrm{K}$ at $37^{\circ} \mathrm{C}$ for $25 \mathrm{~min}$, followed by acetylation in freshly prepared $0.25 \%$ acetic anhydride in $0.1 \mathrm{M}$ triethanolamine $\mathrm{pH} 8.0$ for $10 \mathrm{~min}$. The digoxigenin-labeled riboprobes were diluted in mRNA ISH solution (DAKO, Capinteria, CA) to $1 \mu \mathrm{g} / \mathrm{ml}$, denatured at $100^{\circ} \mathrm{C}$ and then added on the tissue sections. The slides were placed in a humid chamber and incubated in an incubator at $50^{\circ} \mathrm{C}$ overnight for hybridization. After hybridization, the sections were washed to remove the unbound probes and incubated with rabbit HRP-anti-digoxigenin antibody (1:150 dilution) (DAKO) in blocking buffer for $1 \mathrm{~h}$ at room temperature, followed by a wash to remove unbound antibody. The signals were amplified with DAKO GenPoint kit according to the manufacturer's protocol and finally developed by adding DAB. 


\section{Statistical Analysis}

Comparisons of gene expression between groups in qRT-PCR were performed by one-way ANOVA analysis. The stages of liver fibrosis were correlated with MMP-7 immunohistochemical staining scores by using Spearman rank-correlation coefficient. A $P$-value less than 0.05 was regarded as statistically significant.

\section{Results}

\section{The Fibrosis Stage of the Liver Tissues for qRT-PCR Study}

Histological sections with Masson's trichrome stain of the livers in the Control group revealed that the livers were all devoid of fibrosis (stage 0). The stages of liver fibrosis in the Kasai's Procedure group for
qRT-PCR analysis were two of stage I fibrosis, five of stage II and five of stage III. On the other hand, the stages of liver fibrosis in the Liver Transplantation group were three of stage III fibrosis and nine of stage IV fibrosis (Figure 1).

\section{MMP-7 was Overexpressed in the Progress of Liver Fibrosis and Elevated Earlier than MMP-2}

Among the four MMPs studied by the qRT-PCR analysis, we found that only $M M P-2$ and $M M P-7$ expressions significantly elevated in the progress of liver fibrosis associated with biliary atresia (Figure 2). The relative expression level of mRNA evaluated by qRT-PCR revealed that the $M M P-2$ was significantly higher in Liver Transplantation (mean= 13.33) than both in Kasai's Procedure (mean $=5.11)$ $(P=0.001)$ and in Control $($ mean $=5.17)(P=0.010)$. The difference of $M M P-2$ expression between Kasai's
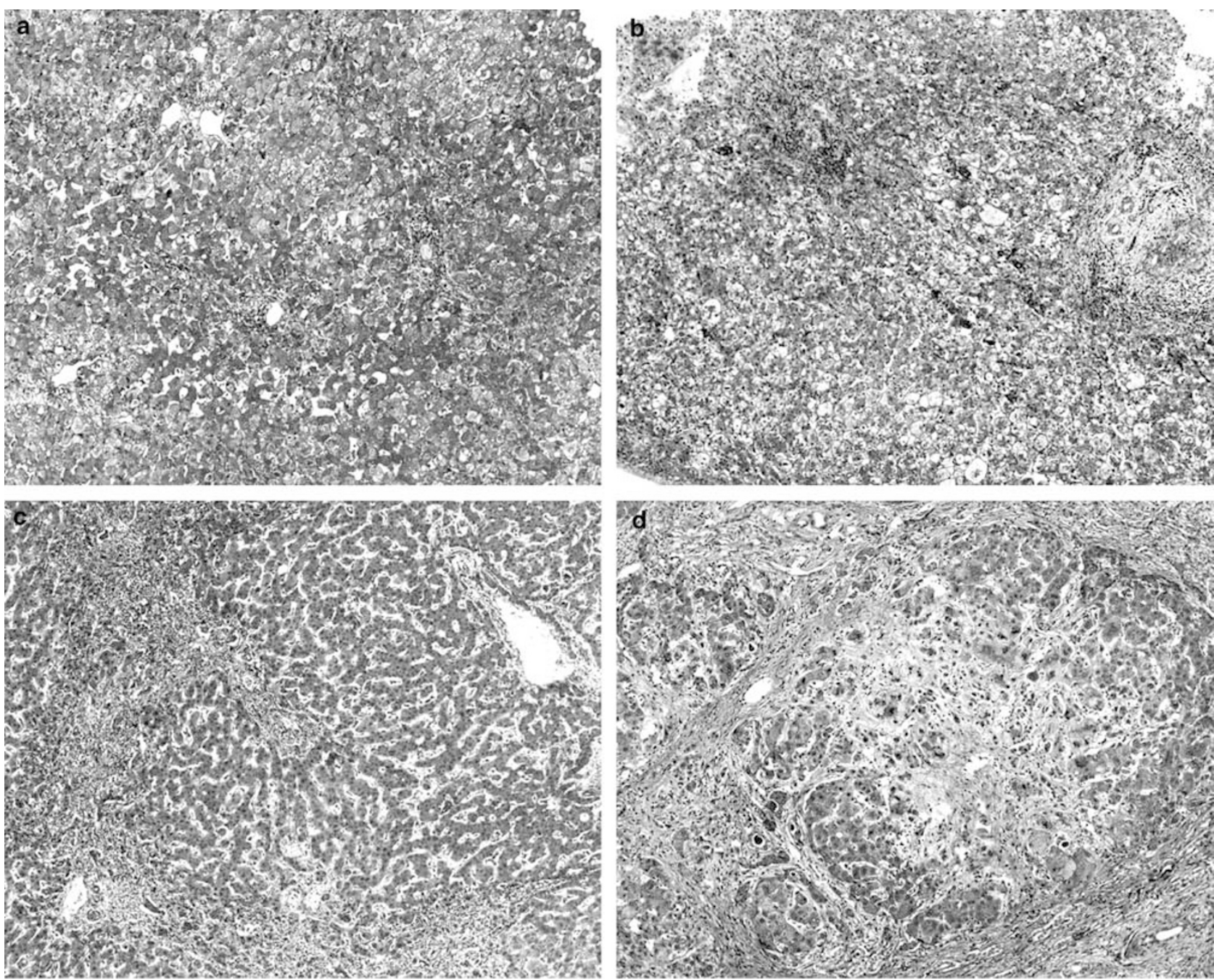

Figure 1 The stages of fibrosis of the representative liver samples. (a) The liver in the Control group shows no fibrosis (stage 0). (b) The liver in the Kasai's Procedure group shows stage I fibrosis characterized by enlarged portal zones. (c) Another liver in the Kasai's Procedure group shows stage III fibrosis characterized by distorted architecture with bridging fibrosis. (d) The liver in the Liver Transplantation group shows stage IV fibrosis characterized by advanced cirrhosis. Masson's trichrome stain; original magnification, $\times 100$. 

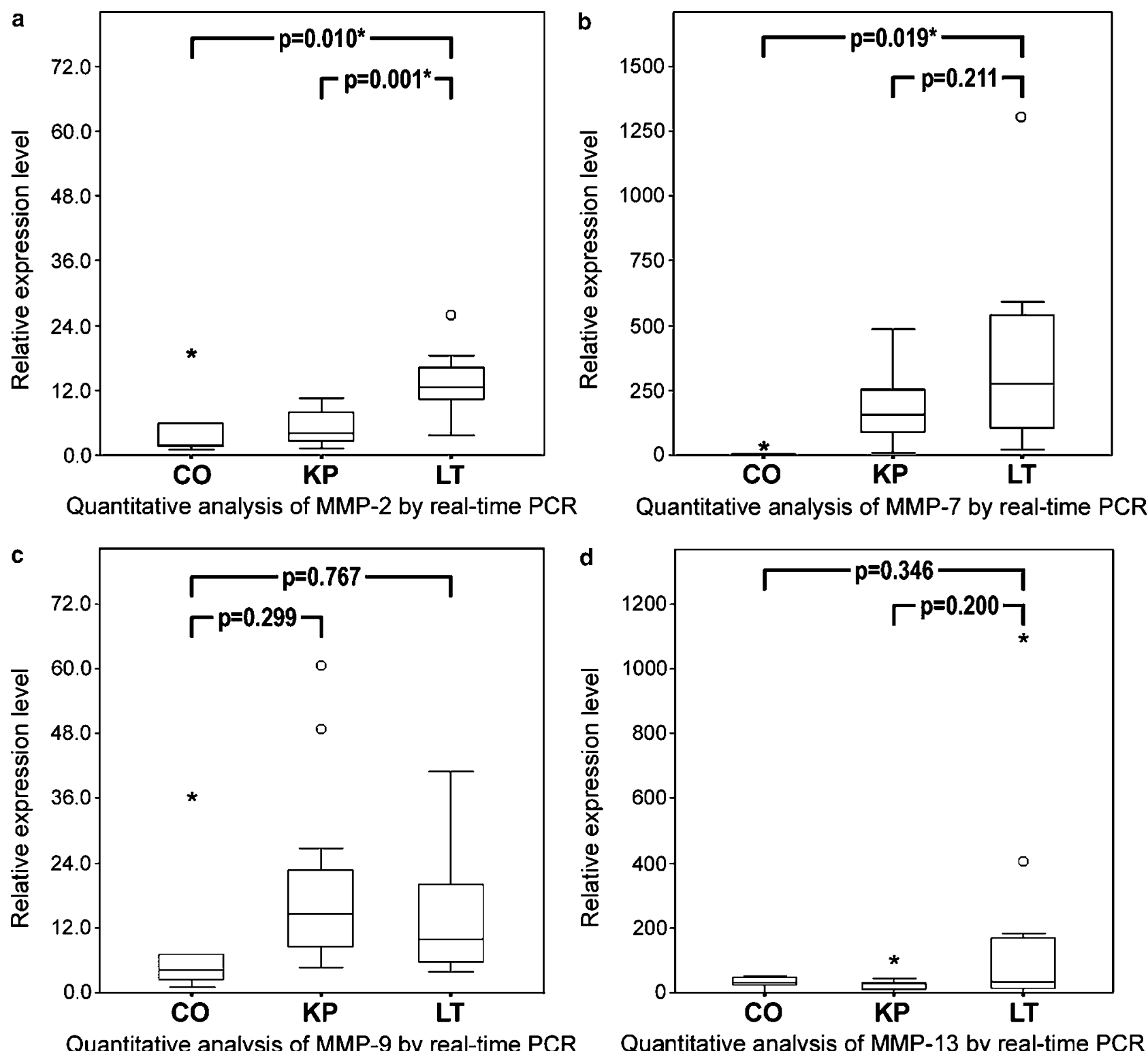

Figure 2 Quantitative analysis of $M M P-2,-7,-9$ and -13 expression by real-time qRT-PCR. (a) $M M P-2$ expression is significantly higher in Liver Transplantation than both in Kasai's Procedure and in Control, but the difference between Kasai's Procedure and Control is not significant. (b) MMP-7 expression shows a sequential elevation from Control, Kasai's Procedure to Liver Transplantation and the difference of MMP-7 expression between Liver Transplantation and Control is significant. (c) MMP-9 expression and (d) MMP-13 expression show no significant difference between groups. $\mathrm{O}$, outlying value; *, extreme outlying value. CO, Control; KP, Kasai's Procedure; LT, Liver Transplantation.

Procedure and Control was not significant $(P>0.999)$. It implied that $M M P-2$ overexpression could be a late event in the progress of liver fibrosis associated with biliary atresia. However, the relative level of expression of MMP-7 was sequentially elevated when comparing Control (mean $=6.35)$, Kasai's Procedure (mean $=193.46$ ) and Liver Transplantation $($ mean $=371.94)$, and there was significant $(P=$ 0.019) difference when comparing Control and Liver Transplantation. Although only the difference of $M M P-7$ expression between Liver Transplantation and Control was significant $(P=0.019)$, the result demonstrated a trend of increase in MMP-7 asso- ciated with the progression of liver fibrosis that might be earlier than the increment of MMP-2. Moreover, the fold difference between groups in $M M P-7$ was much higher than in $M M P-2$. The results were further confirmed with agarose gel electrophoresis and the protein expression of MMP-7 was also verified with Western blotting (Figure 3). Age and sex were obviously not contributing factors to the difference, as the age of the patients in Liver Transplantation group ranked in between those in Control and Kasai's Procedure groups and the sex distributions were relatively homogenous among groups. 
946

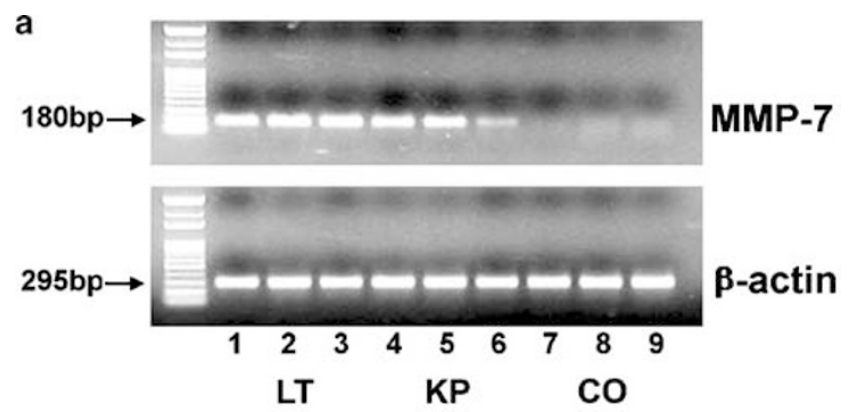

b

CO 2 KP $5^{\text {LT }}$

$30 \mathrm{kd}$ 1

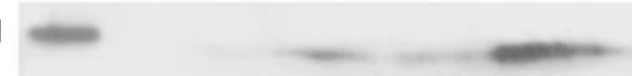

$20 \mathrm{kd}$

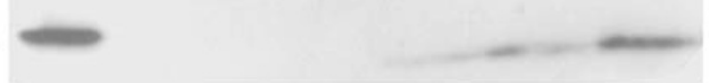

Anti-MMP-7 (Ab-4) Antibody

$30 \mathrm{kd}$

$20 \mathrm{kd}$

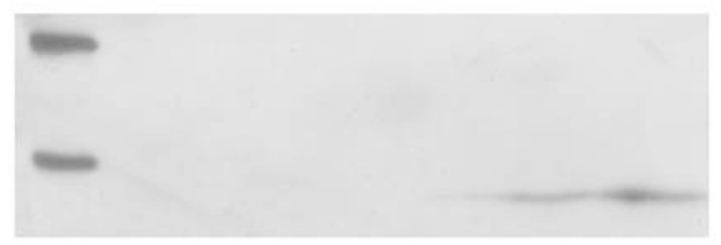

Anti-MMP-7 (Ab-2) Antibody

alpha-tubulin

Figure 3 Verification of MMP-7 expression in biliary atresia. (a) Gel electrophoresis of PCR products after 30 cycles of amplification demonstrates a 180-bp $M M P-7$ band in all cases. The intensity of the $M M P-7$ bands of three representative patients of each group in Liver Transplantation (lanes 1-3) is stronger than those in Kasai's Procedure (lanes 4-6), and the MMP-7 expression in Control (lanes 7-9) is very weak. The intensity of the 295-bp $\beta$ actin band is the same in all patients. (b) Western blotting with rabbit polyclonal antibody $\mathrm{Ab}-4$ recognizing both $28-\mathrm{kDa}$ proenzyme and $18-\mathrm{kDa}$ active enzyme of MMP-7 (upper panel) demonstrates distinct bands of proenzyme in Kasai's Procedure (lanes 3 and 4) and in one of Liver Transplantation (lane 5), and of active enzyme in one of Kasai's Procedure (lane 4) and in both of Liver Transplantation (lanes 5 and 6). No MMP-7 protein is identified in Control (lanes 1 and 2). The protein amounts in Liver Transplantation are more than that in Kasai's Procedure. Western blotting with mouse monoclonal antibody Ab-2 recognizing 18$\mathrm{kDa}$ active enzyme of MMP-7 only (middle panel) demonstrates a band of active MMP-7 in one of Kasai's Procedure (lane 4) and in both of Liver Transplantation (lane 5 and 6) that is consistent with that with Ab-4 antibody. The loading amount of total protein verified by $\alpha$-tubulin (lower panel) is almost the same in all cases. CO, Control; KP, Kasai’s Procedure; LT, Liver Transplantation.

\section{Increased MMP-7 Immunostaining in Kupffer Cells and in Bile Ductules Coincides with Progress of Liver Fibrosis}

To further characterize the MMP-7 protein expression in the liver during the progress of liver fibrosis in biliary atresia, immunohistochemical staining was carried out (Figure 4). It was found that in the
Control group, mild MMP-7 immunoreactivity was present in the cytoplasm of hepatocytes, bile ductular epithelial cells and some nonparenchymal cells morphologically identical to Kupffer cells. In the Kasai's Procedure group, the staining intensity for MMP-7 was increased in all these cells. In the Liver Transplantation group, the MMP-7 immunostaining became more conspicuous not only in hepatocytes, bile ductular epithelial cells and Kupffer cells but also in the interstitial fibrous tissue. Notably, the bile plugs in the bile ductules were remarkably stained with the MMP-7 antibody in both the Kasai's Procedure and Liver Transplantation groups.

In order to elucidate whether the intensity of MMP-7 immunostaining in the liver correlated with the stage of liver fibrosis based on Masson's trichrome stain, 57 archival liver tissues of patients with biliary atresia were analyzed. In the 36 liver tissues of Kasai's procedure, 10 revealed stage III fibrosis, 17 stage II, seven stage I, and two stage 0 . In the 21 liver explants of liver transplantation, 18 revealed stage IV fibrosis and three stage III. Statistical analysis revealed a significant positive correlation of the scores of MMP-7 immunostaining with the stages of liver fibrosis (Spearman's $\sigma=0.512, P<0.001$ ) (Table 2).

\section{ISH Study Localizes MMP-7 Mainly in Hepatocytes, Kupffer Cells and Bile Ductular Epithelial Cells}

ISH was used to localize $M M P-7$ transcript in the liver tissue of biliary atresia. In the Control group, the signals of MMP-7 mRNA were very weak and were localized mainly in the hepatocytes, Kupffer cells, endothelial cells and bile ductular epithelial cells. With progression of liver fibrosis, the staining intensity in the hepatocytes and bile ductular epithelial cells was also increased. The increase in $M M P-7$ signal intensity was most prominent in the bile ductular epithelial cells in the Liver Transplantation group, which was stronger than that in the hepatocytes. Besides, distinct signals were present in Kupffer cells in both the Kasai's Procedure and Liver Transplantation groups. Other cells in the interstitial tissue were only faintly or not stained (Figure 5).

\section{Discussion}

This study reveals that MMP-7 expression is positively related to the progression of liver fibrosis in biliary atresia demonstrated in mRNA level by qRT-PCR and in protein level by immunohistochemistry. The limited cases analyzed by Western blotting also show such a trend. As we did not have enough fresh liver tissues from the other cases to confirm the results of western blotting, we have recently performed ELISA with Quantikine ${ }^{\circledR}$ Human MMP-7 Immunoassay (R\&D Systems) on the liver tissues of 

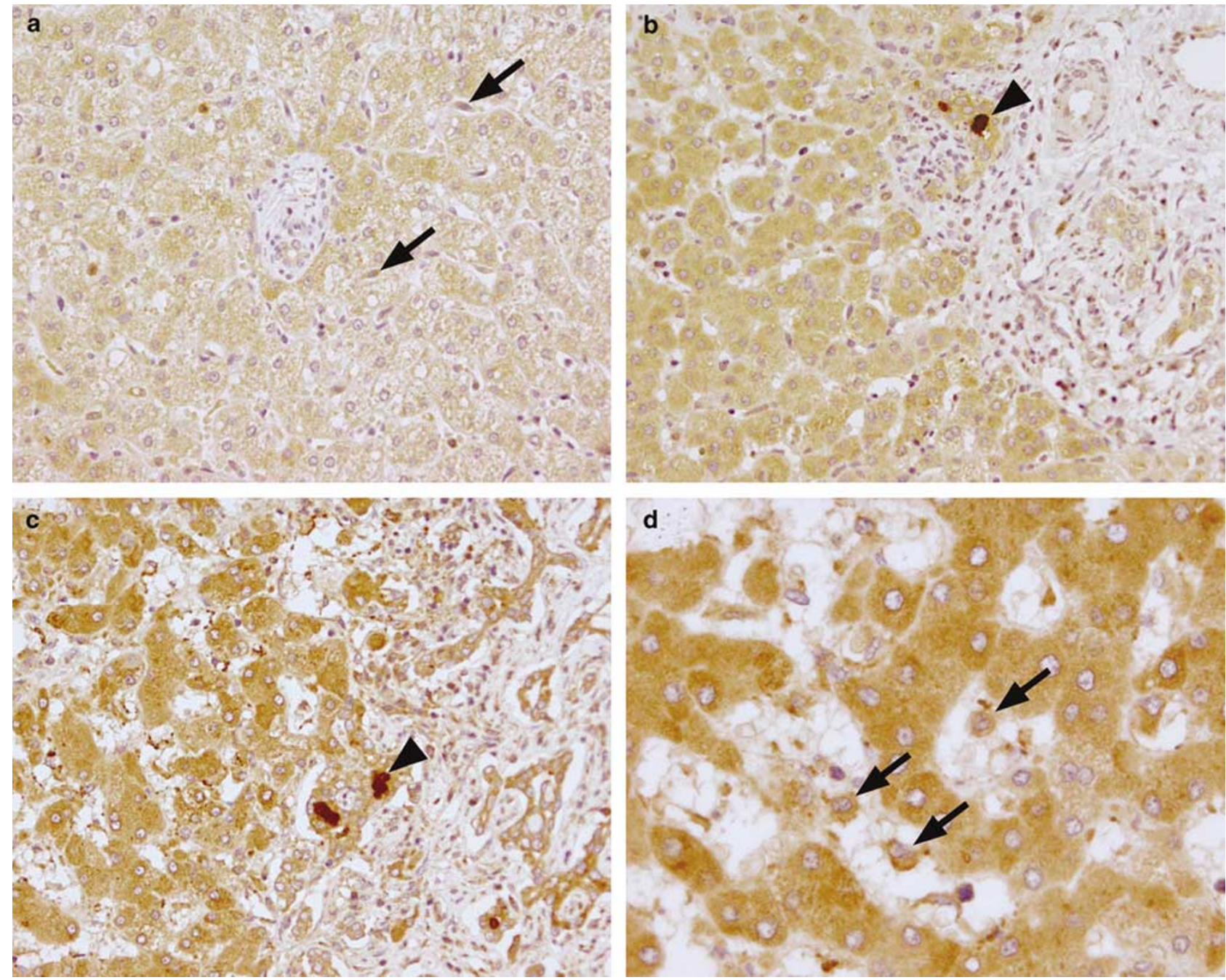

Figure 4 Immunohistochemical study of MMP-7 protein in the liver tissue. (a) In Control, faint (scoring as $1+$ ) immunostaining is noted in hepatocytes, bile ductular epithelial cells, and some nonparenchymal cells morphologically identical to Kupffer cells (arrow). (b) In the representative case of Kasai's Procedure with stage I fibrosis, moderate immunoreactivity for MMP-7 is present (scoring as $2+$ ). In addition to hepatocytes, bile ductular epithelial cells and Kupffer cells, the bile plugs in some bile ductules are strongly stained by MMP7 (arrowhead). (c) In the representative case of Liver Transplantation with stage IV fibrosis, hepatocytes, bile ductular epithelial cells, Kupffer cells and the interstitial fibrous tissue are all strongly stained with MMP-7 antibody (scoring as $3+$ ). The bile plugs are also stained as that in the Kasai's Procedure (arrowhead). (d) High magnification of (c) demonstrates the nonparenchymal cells positive for MMP-7 are morphologically identical to Kupffer cells (arrow). Original magnification, a-c, $\times 200 ; \mathbf{d}, \times 400$.

Table 2 Intensity of MMP-7 immunostaining vs stage of liver fibrosis $^{\mathrm{a}}$

\begin{tabular}{lrrrrr}
\hline MMP-7 staining & 0 & + & ++ & +++ & Total \\
\hline Fibrosis & & & & & \\
0 & 1 & 0 & 1 & 0 & 2 \\
I & 0 & 5 & 2 & 0 & 7 \\
II & 0 & 8 & 8 & 1 & 17 \\
III & 2 & 4 & 3 & 4 & 13 \\
IV & 0 & 2 & 5 & 11 & 18 \\
Total & 3 & 19 & 19 & 16 & 57 \\
\hline
\end{tabular}

${ }^{\mathrm{a}}$ Spearman's $\sigma=0.512, P<0.001$, correlating MMP-7 with fibrosis.

12 new cases (six Kasai's Procedure and six Liver Transplantation). The results revealed that the mean of MMP-7 in Kasai's Procedure group was $5.23 \mathrm{ng}$ per mg total protein and that in Liver Transplantation group was $17.73 \mathrm{ng}$ per mg total protein. The statistical significance came out to be borderline $(P=0.057)$ by $t$-test. Although most of the cases were not included in the present study, the result of ELISA analysis was still consistent with the findings in qRT-PCR and Western blotting.

The histologic features of biliary atresia include cholestasis, periportal ductular reaction and the presence of bile plugs. Periportal/perilobular fibrosis is progressive and eventually causes liver cirrhosis. ${ }^{29}$ Ductular reaction is a common phenomenon in extrahepatic obstruction. The epithelial component of the ductular reaction may derived either from the cells preexisting in the biliary tree, from the circulation or from biliary metaplasia of hepatocytes. ${ }^{30}$ Besides, activated Kupffer cells plays 

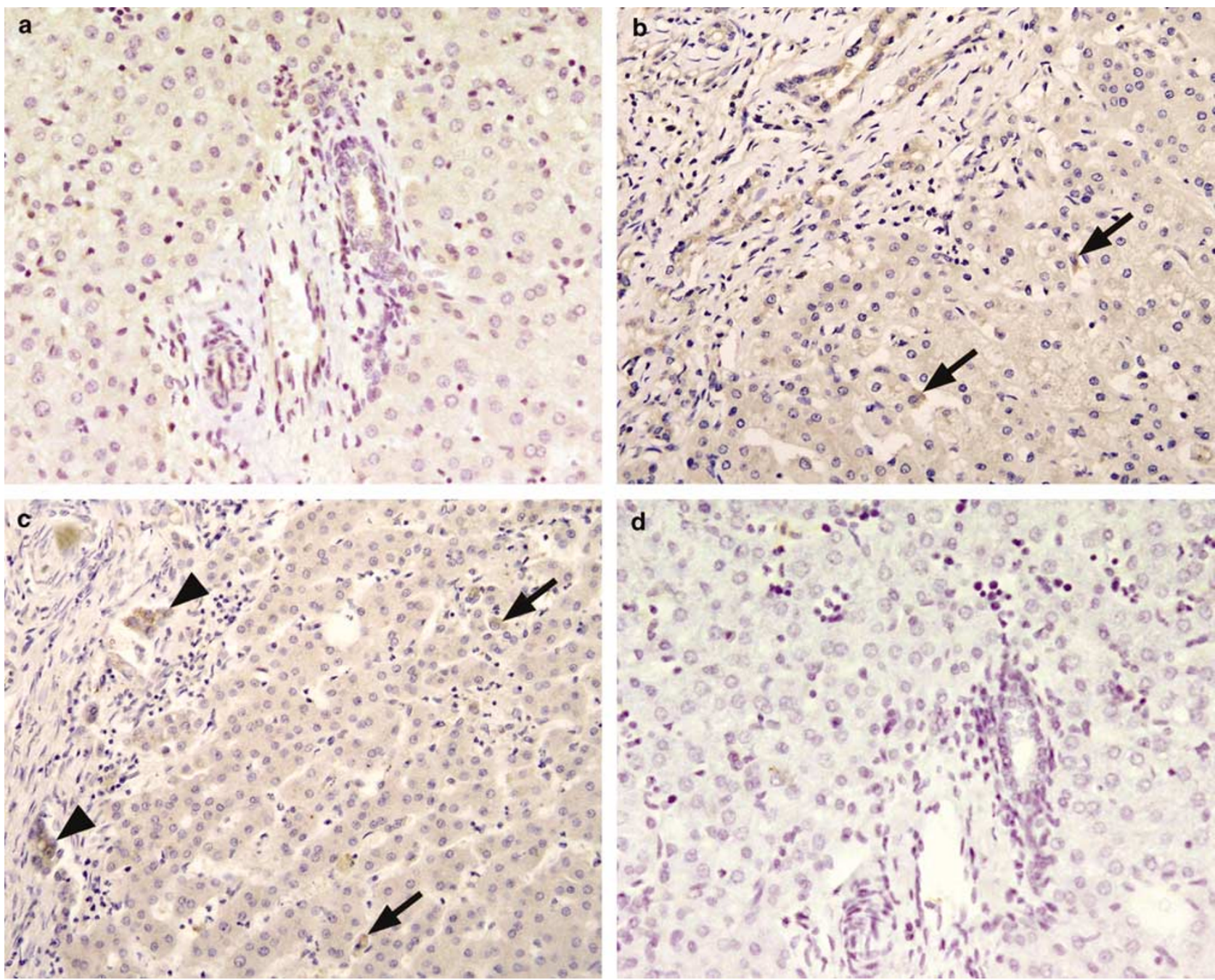

Figure 5 ISH analysis of MMP-7 mRNA in the liver tissue. (a) In Control, very faint signals in hepatocytes, bile ductular epithelial cells, Kupffer cells and endothelial cells are noted. (b) In Kasai's Procedure, the staining intensity is increased mainly in hepatocytes, Kupffer cells (arrows) and bile ductular epithelial cells. (c) In Liver Transplantation, the signals become more prominent in the bile ductular epithelial cells (arrowheads) than in the hepatocytes. (d) A negative control with sense probes shows no signal at all. Original magnification, $\times 200$.

an important role in liver fobrosis by the production of fibrogenic cytokine transforming growth factor $\beta 1$ and MMPs such as MMP-9. ${ }^{31}$ However, the role of MMP-7 in liver fibrosis has not been documented. MMP-7, also known as matrilysin, is the smallest known MMP that lacks the COOH-terminal hemopexin-like domain, which may restrict its substrate specificity. ${ }^{32}$ We detected $M M P-7$ mRNA and protein in the hepatocytes, bile ductular epithelial cells and Kupffer cells. It was consistent with the previous reports that MMP-7 expresses in a few normal human cell types including endometrium, various exocrine glandular epithelia and mononuclear phagocytes..$^{3-35}$ The expression is weak in the normal liver and is highly upregulated in response to progressive liver fibrosis. Such a trend is similar to a recent study of idiopathic pulmonary fibrosis by using oligonucleotide microarrays, which identified
MMP-7 as a key regulator of pulmonary fibrosis in mice and humans. ${ }^{23}$

Just like preferential release of MMP-7 to the airway lumen in lung injury, the bile plugs in the lumens of the bile ductules in patients with liver cirrhosis are strongly positive for MMP-7. It was proposed that luminal MMP-7 may facilitate epithelial cell migration and re-epithelialization in response to lung injury. ${ }^{36}$ MMP-7 was also found to function in intestinal mucosal defense by regulating the activity of defensins and its bactericidal activity. ${ }^{37}$ Taken together, release of MMP-7 from the bile ductular epithelial cells to the lumen in cirrhotic liver may imply a role in tissue repair processes. Furthermore, MMP-7 was found to be able to cleave extracellular matrix and basement membrane proteins such as fibronectin, collagen type IV, and laminin, ${ }^{35}$ and to mediate E-cadherin ectodomain 
shedding in injured lung epithelium and accelerate cell migration. ${ }^{38}$ Hence, the role of MMP-7 in remodeling of extracellular matrix during liver fibrogenesis may require further investigation.

There have been few studies reporting the increase in $M M P-7$ mRNA expression in biliary atresia. Bezerra et al showed a 4.6-fold change in hepatic MMP-7 in patients with biliary atresia comparing to those with neonatal intrahepatic cholestasis by cRNA hybridization against oligonucleotide-based gene chips. ${ }^{24}$ Chen et $a l^{25}$ demonstrated a similar result that MMP-7 expression in biliary atresia liver had 2.46-fold change over that in normal liver and 1.93-fold change over diseased controls by cDNA microarray. Notably, $M M P-7$ was the only MMP significantly upregulated in the liver of biliary atresia in these two studies. Besides the role in pulmonary fibrosis mentioned above, $M M P-7$ has also been found to be related to progression of tubulointerstitial fibrosis in renal tubular injury in human, ${ }^{39}$ and to be involved in the tissue remodeling process and fibrosis of the left atria during progression of heart failure in rat. ${ }^{40}$ Furthermore, Lichtinghagen et $a l^{17}$ analyzed hepatic mRNA expression and reported that $M M P-2, M M P-7$, and TIMP-1 provided the best discrimination between cirrhosis and pre-cirrhotic stages in patients with chronic active hepatitis $\mathrm{C}$, and steadily increasing mRNA expression with disease progression in MMP$1,-2,-7$ and -14 closely correlated to the parameters of fibrogenesis in hepatitis $\mathrm{C}$ virus-induced liver cirrhosis. ${ }^{41}$ These previous reports support our findings that MMP-7 is a major MMP associated with liver fibrosis, and our study is the first report to correlate the stage of liver fibrosis with MMP-7 expression in biliary atresia patients.

MMP-2, known as a $72 \mathrm{kDa}$ gelatinase, can degrade gelatin, fibronectin, laminin and nidogen. $^{31,42}$ It has been reported that hepatic $M M P-2$ mRNA expression was increased in fibrotic liver caused by hepatobiliary disorders including biliary atresia, ${ }^{20}$ and serum MMP-2 level was higher in biliary atresia compared with the control. ${ }^{15}$ However, Walsh et al ${ }^{18}$ reported that the serum levels of MMP-2 failed to correlate with fibrosis and with histological index in the patients with chronic hepatitis C. These findings, together with the studies of Lichtinghagen et $a l^{17,41}$ on hepatitis C-related liver fibrosis, agreed with our result that $M M P-2$ was significantly upregulated in the stage of advanced cirrhosis but not in the early stage in biliary atresia. It implies that increase in MMP-2 expression in liver could be a late event in the progress of liver cirrhosis but not necessary to be biliary atresia-related.

In conclusion, MMP-7 is identified as a major MMP increased in expression during the progress of liver fibrosis in biliary atresia. Downregulation of MMP-7 might be able to influence the course of tissue remodeling and to change the outcome of liver fibrosis. Further characterization with the use of knockout mice could be useful to verify the findings and to develop an antifibrotic strategy.

\section{Acknowledgements}

We thank Dr Chie-Song Hsieh and Dr Shin-Ye Lee for providing the samples used in this study and Dr Chao-Hung Lee, Department of Pathology and Laboratory Medicine, Indiana University School of Medicine, for critical editing of this manuscript. This study is supported by grants from the National Science Council (NSC 91-2314-B-182-065 and NSC 92-2321-B-182A-001) and the Chang Gung Memorial Hospital (CMRP G8005), Taiwan.

\section{References}

1 Perlmutter DH, Shepherd RW. Extrahepatic biliary atresia: a disease or a phenotype? Hepatology 2002;35: 1297-1304.

2 Chen CL, Chen YS, de Villa VH, et al. Minimal blood loss living donor hepatectomy. Transplantation 2000; 69:2580-2586.

3 Jain A, Reyes J, Kashyap R, et al. Long-term survival after liver transplantation in 4,000 consecutive patients at a single center. Ann Surg 2000;232:490-500.

4 Miller CM, Gondolesi GE, Florman S, et al. One hundred nine living donor liver transplants in adults and children: a single-center experience. Ann Surg 2001;234:301-311 discussion 11-12.

5 Bates MD, Bucuvalas JC, Alonso MH, et al. Biliary atresia: pathogenesis and treatment. Semin Liver Dis 1998;18:281-293.

6 Friedman SL, Maher JJ, Bissell DM. Mechanisms and therapy of hepatic fibrosis: report of the AASLD Single Topic Basic Research Conference. Hepatology 2000;32: 1403-1408.

7 Benyon RC, Arthur MJ. Extracellular matrix degradation and the role of hepatic stellate cells. Semin Liver Dis 2001;21:373-384.

8 Faiz Kabir Uddin Ahmed A, Ohtani H, Nio M, et al. In situ expression of fibrogenic growth factors and their receptors in biliary atresia: comparison between early and late stages. J Pathol 2000;192:73-80.

9 Ahmed AF, Nio M, Ohtani $\mathrm{H}$, et al. Clinical significance of c-kit expression in biliary atresia. Pediatr Surg Int 2001;17:601-603.

10 Liu C, Chiu JH, Chin T, et al. Expression of aminopeptidase $\mathrm{N}$ in bile canaliculi: a predictor of clinical outcome in biliary atresia and a potential tool to implicate the mechanism of biliary atresia. J Surg Res 2001;100:76-83.

11 Davenport M, Gonde C, Redkar R, et al. Immunohistochemistry of the liver and biliary tree in extrahepatic biliary atresia. J Pediatr Surg 2001;36:1017-1025.

12 Rosensweig JN, Omori M, Page K, et al. Transforming growth factor-beta1 in plasma and liver of children with liver disease. Pediatr Res 1998;44:402-409.

13 Overall CM, Lopez-Otin C. Strategies for MMP inhibition in cancer: innovations for the post-trial era. Nat Rev Cancer 2002;2:657-672.

14 Cursio R, Mari B, Louis K, et al. Rat liver injury after normothermic ischemia is prevented by a phosphinic 
matrix metalloproteinase inhibitor. FASEB J 2002;16: 93-95.

15 Kobayashi H, Li ZX, Yamataka A, et al. Clinical evaluation of serum levels of matrix metalloproteinases and tissue inhibitors of metalloproteinases as predictors of progressive fibrosis in postoperative biliary atresia patients. J Pediatr Surg 2002;37: 1030-1033.

16 Boeker KH, Haberkorn CI, Michels D, et al. Diagnostic potential of circulating TIMP-1 and MMP-2 as markers of liver fibrosis in patients with chronic hepatitis C. Clin Chim Acta 2002;316:71-81.

17 Lichtinghagen R, Michels D, Haberkorn CI, et al. Matrix metalloproteinase (MMP)-2, MMP-7, and tissue inhibitor of metalloproteinase-1 are closely related to the fibroproliferative process in the liver during chronic hepatitis C. J Hepatol 2001;34:239-247.

18 Walsh KM, Timms P, Campbell S, et al. Plasma levels of matrix metalloproteinase-2 (MMP-2) and tissue inhibitors of metalloproteinases-1 and -2 (TIMP-1 and TIMP-2) as noninvasive markers of liver disease in chronic hepatitis C: comparison using ROC analysis. Dig Dis Sci 1999;44:624-630.

19 Vaillant B, Chiaramonte MG, Cheever AW, et al. Regulation of hepatic fibrosis and extracellular matrix genes by the th response: new insight into the role of tissue inhibitors of matrix metalloproteinases. J Immunol 2001;167:7017-7026.

20 Benyon RC, Iredale JP, Goddard S, et al. Expression of tissue inhibitor of metalloproteinases 1 and 2 is increased in fibrotic human liver. Gastroenterology 1996;110:821-831.

21 Herbst H, Wege T, Milani S, et al. Tissue inhibitor of metalloproteinase-1 and -2 RNA expression in rat and human liver fibrosis. Am J Pathol 1997;150: 1647-1659.

22 Schuppan D, Ruehl M, Somasundaram R, et al. Matrix as a modulator of hepatic fibrogenesis. Semin Liver Dis 2001;21:351-372.

23 Zuo F, Kaminski N, Eugui E, et al. Gene expression analysis reveals matrilysin as a key regulator of pulmonary fibrosis in mice and humans. Proc Natl Acad Sci USA 2002;99:6292-6297.

24 Bezerra JA, Tiao G, Ryckman FC, et al. Genetic induction of proinflammatory immunity in children with biliary atresia. Lancet 2002;360:1653-1659.

25 Chen L, Goryachev A, Sun J, et al. Altered expression of genes involved in hepatic morphogenesis and fibrogenesis are identified by cDNA microarray analysis in biliary atresia. Hepatology 2003;38:567-576.

26 Ferrell L. Liver pathology: cirrhosis, hepatitis, and primary liver tumors. Update and diagnostic problems. Mod Pathol 2000;13:679-704.

27 Huang CC, Qiu JT, Kashima ML, et al. Generation of type-specific probes for the detection of single-copy human papillomavirus by a novel in situ hybridization method. Mod Pathol 1998;11:971-977.
28 Huang CC, Chuang JH, Huang LL, et al. The human Delta-like 1 homologue is implicated in the progression of liver fibrosis in biliary atresia. J Pathol 2004;202:172-179.

29 Ishak KG, Sharp HL. Developmental abnormalities and liver disease in childhood. In: MacSween RNM, Burt AD, Protmann BC, Ishak KG, Scheuer PJ, Anthony PP (eds). Pathology of the liver, 4th edn. Churchill Livingstone: London, 2002, pp 107-154.

30 Roskams TA, Theise ND, Balabaud C, et al. Nomenclature of the finer branches of the biliary tree: canals, ductules, and ductular reactions in human livers. Hepatology 2004;39:1739-1745.

31 Rojkind M, Greenwel P. Pathophysiology of liver fibrosis. In: Arias IM (ed). The Liver: Biology and Pathobiology, 4th edn. Lippincott Williams \& Wilkins: Philadelphia, 2001, pp 721-738.

32 Birkedal-Hansen $\mathrm{H}$, Moore WG, Bodden MK, et al. Matrix metalloproteinases: a review. Crit Rev Oral Biol Med 1993;4:197-250.

33 Saarialho-Kere UK, Crouch EC, Parks WC. Matrix metalloproteinase matrilysin is constitutively expressed in adult human exocrine epithelium. J Invest Dermatol 1995;105:190-196.

34 Busiek DF, Baragi V, Nehring LC, et al. Matrilysin expression by human mononuclear phagocytes and its regulation by cytokines and hormones. J Immunol 1995;154:6484-6491.

35 Wilson CL, Matrisian LM. Matrilysin: an epithelial matrix metalloproteinase with potentially novel functions. Int J Biochem Cell Biol 1996;28:123-136.

36 Dunsmore SE, Saarialho-Kere UK, Roby JD, et al. Matrilysin expression and function in airway epithelium. J Clin Invest 1998;102:1321-1331.

37 Wilson CL, Ouellette AJ, Satchell DP, et al. Regulation of intestinal alpha-defensin activation by the metalloproteinase matrilysin in innate host defense. Science 1999;286:113-117.

38 McGuire JK, Li Q, Parks WC. Matrilysin (matrix metalloproteinase-7) mediates E-cadherin ectodomain shedding in injured lung epithelium. Am J Pathol 2003;162:1831-1843.

39 Surendran K, Simon TC, Liapis H, et al. Matrilysin (MMP-7) expression in renal tubular damage: association with Wnt4. Kidney Int 2004;65:2212-2222.

40 Boixel C, Fontaine V, Rucker-Martin C, et al. Fibrosis of the left atria during progression of heart failure is associated with increased matrix metalloproteinases in the rat. J Am Coll Cardiol 2003;42:336-344.

41 Lichtinghagen R, Bahr MJ, Wehmeier M, et al. Expression and coordinated regulation of matrix metalloproteinases in chronic hepatitis $\mathrm{C}$ and hepatitis $\mathrm{C}$ virus-induced liver cirrhosis. Clin Sci (London) 2003; 105:373-382.

42 Westermarck J, Kahari VM. Regulation of matrix metalloproteinase expression in tumor invasion. FASEB J 1999;13:781-792. 